\title{
Validação de equações preditivas da área de secção transversa do tronco
}

\author{
Validation of predictive equations of the cross-sectional area \\ of the human trunk
}

\author{
Jorge Estrela Morais \\ Mário Jorge Costa \\ Jean Erik Mejias ${ }^{1}$ \\ Daniel Almeida Marinho ${ }^{2}$ \\ António José Silva ${ }^{3}$ \\ Tiago Manuel Barbosa ${ }^{1}$
}

1. Instituto Politécnico de Bragança. Departamento de Ciências de Desporto. Centro de Investigação em Desporto, Saúde e Desenvolvimento Humano. Bragança, Portugal.

2. Universidade da Beira Interior. Departamento de Ciências de Desporto. Centro de Investigação em Desporto, Saúde e Desenvolvimento Humano. Covilhã, Portugal.

\section{Universidade de Trás-os-Montes e Alto Douro. Departamento de Ciências de Desporto. Centro de Investigação em Desporto, Saúde e De- senvolvimento Humano. Vila Real, Portugal.}

Recebido em 17/02/11 Revisado em 08/05/11 Aprovado em 18/06/11
Resumo - O objectivo do estudo foi desenvolver e validar equações preditivas da área de secção transversa do tronco humano, Os modelos foram desenvolvidos para o sexo masculino e de acordo com o nível competitivo, A amostra foi composta por 152 sujeitos, todos praticantes de Natação Pura desportiva, com idades entre os 10 e os 32 anos de idade, Para o desenvolvimento dos modelos foi utilizado um grupo de sujeitos para estimar a equação e um outro para validar. Foram avaliadas as seguintes características antropométricas: (i) massa corporal; (ii) estatura; (iii) diâmetro bi-acromial; (iv) diâmetro tóraco-sagital; (v) perímetro peitoral e (vi) área de secção transversal do tronco. Os modelos preditivos foram desenvolvidos através de análise de regressão linear múltipla passo-a-passo e num dos casos, com recurso ao nível competitivo enquanto variável dummy. Todos os modelos desenvolvidos incluíram como variáveis independentes o diâmetro tóraco-sagital e o perímetro peitoral $\left(0,32 \leq \mathrm{R}^{2} \leq 0,48 ; \mathrm{P}<0,01\right)$. No processo de validação entre as áreas de secção transversal do tronco medida e estimada, não se verificaram diferenças significativas entre valores médios $(\mathrm{P}>0,05)$, as regressões lineares simples foram moderadas $\left(0,23 \leq \mathrm{R}^{2} \leq 0,39 ; 0,01 \leq \mathrm{P} \leq\right.$ $0,001)$ e o critério de Bland Altman foi em todos os casos cumprido. Pode concluir-se que os modelos desenvolvidos para nadadores masculinos, e de acordo com o nível competitivo, predizem com validade a área de secção transversa do tronco de nadadores.

Palavras-chave: Atleta; Estudos de validação; Natação; Superfície corporal.

Abstract - The objective of this study was to develop and validate predictive equations of the cross-sectional area of the human trunk. The models were developed for males according to their level of expertise. The sample comprised 152 male subjects, all of them with a background in competitive or recreational swimming. Their ages ranged between 10 and 32 years. Two different groups of subjects were used to estimate and validate the equation. The following anthropometric characteristics were assessed: (i) body weight, (ii) height, (iii) biacromial diameter, (iv) sagittal thoracic diameter, (v) chest circumference, and (vi) cross-sectional area of the trunk. Predictive models were developed using stepwise multiple linear regression analysis. One of the models used level of expertise as a dummy variable. All models included sagittal thoracic diameter and chest circumference as independent variables $\left(0.32 \leq R^{2} \leq 0.48 ; P<0.01\right)$. The validation process of the measured and estimated cross-sectional area of the trunk did not reveal any significant differences between the mean values $(P>0.05)$. The simple linear regressions were moderate $\left(0.23 \leq R^{2} \leq 0.55 ; 0.01 \leq P \leq 0.001\right)$, and the Bland-Altman criterion was met in all cases. Therefore, our findings suggest that the models developed for male swimmers according to their level of expertise are able to provide a valid prediction of the cross-sectional area of the trunk. Key words: Athletes; Body surface area; Swimming; Validation studies. 


\section{INTRODUÇÃO}

$\mathrm{O}$ acto de nadar depende da interacção de um conjunto de forças externas existentes no meio aquático, como sejam a propulsão, o arrasto, a flutuabilidade e o peso'. Durante a locomoção humana, com recurso a técnicas de nado, procura-se optimizar a velocidade, diminuindo o arrasto e aumentando a propulsão. Entende-se por arrasto hidrodinâmico a força de resistência que o nadador tem de vencer de forma a produzir a translação do seu centro de massa ${ }^{1}$.

O arrasto hidrodinâmico é classificado como uma força externa que actua sobre o corpo do nadador, com a mesma direcção e com sentido oposto ao deslocamento, pelo que quanto menor for a sua intensidade, maior será a velocidade de deslocamento, caso todas as outras condições se mantenham constantes ${ }^{2}$. Numericamente, é calculado através da equação de arrasto newtoniano que inclui a área de superfície frontal, logo condicionado pela forma e velocidade do objecto que se desloca no meio aquático ${ }^{3-5}$.

Quer na avaliação do arrasto passivo ${ }^{6}$, quer do arrasto activo ${ }^{7}$ é necessária a inclusão da variável área de secção transversa do tronco (ASTT) do sujeito. A ASTT pode ser medida directamente através de planimetria, tomografia computacional, ressonância magnética ou através de softwares bidimensionais. Ainda é possível a estimação da ASTT através de equações matemáticas ${ }^{8}$.

Considera-se que as equações são susceptíveis de algumas limitações ${ }^{4}$, como sejam: (i) a amostra utilizada para o desenvolvimento do modelo preditivo foi reduzida; (ii) as características antropométricas dos nadadores de então (anos 70) poderão não ser idênticas às que se podem encontrar actualmente; (iii) a capacidade preditiva da equação do estudo de Clarys $^{8}$ não é elevada ( $\mathrm{R}=0,70$ ); (iv) no estudo em causa, a equação nunca foi objecto de validação numa amostra independente. Num outro estudo ${ }^{9}$, foi desenvolvido um modelo de análise de trajectórias para a avaliação da força de arrasto activo em jovens nadadores. $\mathrm{O}$ modelo era baseado em variáveis antropométricas, hidrodinâmicas e biomecânicas, entre as quais a ASTT, No entanto, o modelo excluiu a ASTT, levando os autores a sugerirem o desenvolvimento e validação de diversas equações preditivas de ASTT por sexo, faixa etária e nível competitivo9. Assim, constata-se que as características da composição corporal em atletas podem auxiliar treinadores e demais ajudantes com informações relevantes durante o processo de treino ${ }^{10}$.
Neste sentido, o objectivo do presente estudo foi desenvolver e validar equações preditivas da área de secção transversa do tronco para atletas de natação.

\section{PROCEDIMENTOS METODOLÓGICOS}

\section{Amostra}

A amostragem utilizada foi a de conveniência, visto estar-se restrito a sujeitos praticantes de actividades aquáticas, nomeadamente a Natação Pura Desportiva e à sua autorização (ou dos respectivos encarregados de educação) para a recolha dos dados. A amostra foi composta por 152 sujeitos do sexo masculino. As características gerais da amostra encontram-se descritas na tabela 1. Foram considerados como critérios de inclusão: (i) o sexo (masculino); (ii) a idade (entre os 10 e 32 anos de idade); (iii) isenção de patologias musculó-esqueléticas; (iv) o nível competitivo dos sujeitos. Em relação ao nível competitivo dos sujeitos, os praticantes foram considerados como pertencentes a uma de duas categorias ou grupos de coorte: (i) nadadores com participação regular em provas de Natação de competição organizadas pela Federação Portuguesa de Natação ou quaisquer das suas associações regionais (i,e, experts) ou; (ii) não federados, predominantemente de índole recreativa, mas com pontuais participações em provas de natação pura, abertas a não federados (i,e, não experts). A figura 1 sintetiza a distribuição da amostra total nos diversos grupos de coorte.

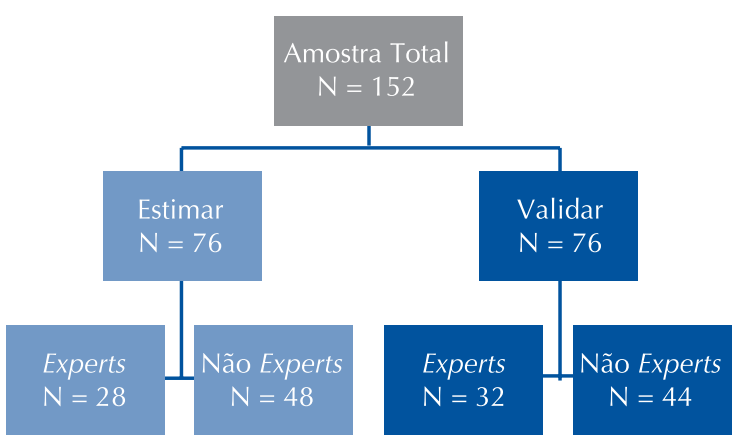

Figura 1. Distribuição da amostra total nos diversos grupos de coorte.

Todos os sujeitos voluntariaram-se para o estudo e eles próprios ou os seus encarregados de educação deram o consentimento. De igual forma, foram respeitados os princípios da Declaração de Helsínquia no que diz respeito a procedimentos científicos, envolvendo Seres Humanos. O Comité de Ética do Instituto Politécnico de Bragança aprovou o estudo. 


\section{Recolha de Dados}

A avaliação da ASTT foi realizada com os sujeitos numa posição bípede, plantígrada e ortostática, com a cabeça numa posição neutra, os membros superiores em extensão completa no prolongamento do corpo com a superfície palmar de uma mão em contacto com o dorso da mão oposta e os dedos também em extensão.

Os sujeitos foram fotografados no plano transversal superior com uma máquina digital (Sony DSC-T7. Tóquio, Japão) de 5,1 mega pixels, junto a um objecto de calibração com 0,945 [m] de largura que se encontrava ao nível do apêndice xifóide. Posteriormente, as imagens foram editadas no software específico para medição de áreas (Universal Desktop Ruler, v3.3.3268, AVPSoft. E.U.A.).

Os procedimentos a efectuar foram: (i) calibração da escala de medição; (ii) delimitação, através de digitalização manual com recurso a um cursor, do maior perímetro do sujeito, ao nível do apêndice xifóide, observável na fotografia; (iii) cálculo e registo da ASTT.

Foram ainda avaliados os seguintes parâmetros antropométricos: (i) massa corporal; (ii) estatura; (iii) diâmetro bi-acromial; (iv) diâmetro tóraco-sagital e; (v) perímetro peitoral. Estas variáveis são recorrentemente descritas em estudos de Natação Pura Desportiva ${ }^{11}$.

Todas as medições foram realizadas com os sujeitos a usarem um fato de banho têxtil e uma touca. A massa corporal foi medida com recurso a uma balança digital (SECA, 884, Hamburgo, Alemanha). A estatura foi avaliada com um estadiómetro digital (SECA, 242, Hamburgo, Alemanha). Os diâmetros bi-acromial e tóraco-sagital foram medidos utilizando-se um clip de medição (Campbell, 20, RossCraft, Canadá), com o avaliador colocado no plano sagital direito relativamente ao sujeito. A medição do perímetro peitoral foi realizada com uma fita métrica (RossCraft, Canadá), estando os sujeitos a simular a posição hidrodinâmica, mas na postura ortostática. As medições foram realizadas por um avaliador experiente. As variáveis foram medidas três vezes, e para posterior análise, foi considerada a média das três avaliações de cada sujeito.

\section{Procedimentos Estatísticos}

Foi realizada a análise de simetria (skewness) e de achatamento (kurtosis) das curvas e aplicado o teste de Kolmogorov-Smirnov para averiguar se os valores registados apresentam uma distribuição normal.

$\mathrm{Na}$ análise descritiva, foram calculados os seguintes parâmetros: (i) média; (ii) um desvio padrão; (iii) o valor mínimo; (iv) o valor máximo e; (v) o coeficiente de variação de todas as variáveis adoptadas.

Os modelos de estimação da ASTT foram desenvolvidos através de regressão linear múltipla passo-a-passo, tendo em consideração a variável endógena (i,e, ASTT) e as variáveis exógenas (i,e, massa corporal, estatura, diâmetro bi-acromial, diâmetro tóraco-sagital e perímetro peitoral). Os modelos foram desenvolvidos para: (i) a totalidade da amostra masculina; (ii) o subgrupo experts; (iii) o subgrupo não experts; (iv) a totalidade da amostra masculina com inclusão do nível competitivo enquanto variável dummy ( 0 = não expert; 1 = expert). Considerou-se que as variáveis independentes entraram no modelo caso $\mathrm{F} \geq 4,0$ e removidas se $\mathrm{F} \leq 3,96^{12}$. Foi tomado como elemento de análise o coeficiente de determinação $\left(R^{2}\right)$ e o coeficiente de determinação ajustado $\left(\mathrm{Ra}^{2}\right)$.

A validação dos modelos foi realizada como sugerido por diversos estudos ${ }^{13-15}$ : (i) a comparação dos valores médios; (ii) o coeficiente de determinação e; (iii) o gráfico de Bland-Altman. Numa primeira fase, compararam-se os valores médios entre a área de secção transversa medida e a estimada através dos modelos desenvolvidos com recurso ao Teste T-student Emparelhado $(\mathrm{p} \leq 0,05)$. Considerou-se a análise de modelos de regressão linear simples entre as áreas de secção transversa medida e estimada. Foi tomado como elemento de análise o coeficiente de determinação e o coeficiente de determinação ajustado. Para uma análise qualitativa, foi definido que a relação era: (i) muito fraca se $\mathrm{R}^{2}<0,04$; (ii) fraca se $0,04 \leq \mathrm{R}^{2}<0,16$; (iii) moderada se $0,16 \leq$ $\mathrm{R}^{2}<0,49$; (iv) elevada se $0,49 \leq \mathrm{R}^{2}<0,81$; (v) muito elevada se $0,81 \leq R^{2} \leq 1,0$. Por último, foi produzido o plot de Bland-Altman ${ }^{16}$, que analisa a concordância entre diferentes tipos de medição, explicando se estes métodos terão uma boa correlação quando um conjunto de amostras é escolhido de tal modo que a variável a ser determinada varia muito entre eles. Considerou-se a validação satisfatória dos modelos se $80 \%$ dos plots entre a área de secção transversa medida e a estimada se encontrarem dentro dos 95 $\%$ do intervalo de confiança, correspondentes a \pm 1,96 desvios padrão ${ }^{18}$.

\section{RESULTADOS}

As tabelas 1, 2 e 3 apresentam as estatísticas descritivas das variáveis antropométricas avaliadas para a totalidade da amostra, para o subgrupo masculino experts e para o subgrupo masculino não experts, respectivamente. 
Tabela 1. Caracterização antropométrica da totalidade da amostra.

\begin{tabular}{lcccccc}
\hline & MC $(\mathrm{kg})$ & Estatura $(\mathrm{cm})$ & DBC $(\mathrm{cm})$ & DTS $(\mathrm{cm})$ & PP $(\mathrm{cm})$ & ASTT $\left(\left[\mathrm{cm}^{2}\right)\right.$ \\
\hline Média & 63,61 & 169,41 & 35,41 & 22,43 & 86,90 & 747,46 \\
1 DP & 15,10 & 12,12 & 5,07 & 3,00 & 9,31 & 184,59 \\
Mínimo & 28,00 & 134,00 & 19,90 & 11,50 & 61,50 & 373,59 \\
Máximo & 108,60 & 189,00 & 50,50 & 31,00 & 112,00 & 1371,00 \\
CV & 23,74 & 7,15 & 14,32 & 13,37 & 10,71 & 24,70 \\
\hline
\end{tabular}

$\mathrm{MC}=$ massa corporal; $\mathrm{DBC}$ = diâmetro bi-acromial; DTS = diâmetros tóraco-sagital; PP = perímetro do peito; ASTT = área de secção transversa do tronco

Tabela 2. Caracterização antropométrica do subgrupo de experts.

\begin{tabular}{lcccccc}
\hline & MC $(\mathrm{kg})$ & Estatura $(\mathrm{cm})$ & DBC $(\mathrm{cm})$ & DTS $(\mathrm{cm})$ & PP $(\mathrm{cm})$ & ASTT $\left(\left[\mathrm{cm}^{2}\right)\right.$ \\
Média & 54,83 & 164,52 & 37,46 & 22,44 & 81,63 & 715,57 \\
1 DP & 11,78 & 11,73 & 6,34 & 3,72 & 7,49 & 175,51 \\
Mínimo & 32,00 & 141,00 & 19,90 & 11,50 & 64,00 & 417,46 \\
Máximo & 86,00 & 188,40 & 50,50 & 31,00 & 100,00 & 1371,00 \\
CV & 21,48 & 7,12 & 16,92 & 16,57 & 9,17 & 24,52 \\
\hline
\end{tabular}

$\mathrm{MC}=$ massa corporal; $\mathrm{DBC}=$ diâmetro bi-acromial; DTS = diâmetros tóraco-sagital; $\quad \mathrm{PP}=$ perímetro do peito; ASTT = área de secção transversa do tronco.

Tabela 3. Caracterização antropométrica do subgrupo de não experts.

\begin{tabular}{lcccccc}
\hline & MC $(\mathrm{kg})$ & Estatura $(\mathrm{cm})$ & DBC $(\mathrm{cm})$ & DTS $(\mathrm{cm})$ & PP $(\mathrm{cm})$ & ${\text { ASTT }\left(\left[\mathrm{cm}^{2}\right)\right.}$ \\
\hline Média & 69,07 & 172,50 & 34,12 & 22,43 & 90,23 & 768,48 \\
1 DP & 14,38 & 11,38 & 3,53 & 2,47 & 8,81 & 188,34 \\
Mínimo & 28,00 & 134,00 & 23,80 & 15,40 & 61,50 & 373,59 \\
Máximo & 108,60 & 189,00 & 40,20 & 30,10 & 112,00 & 1366,66 \\
CV & 20,81 & 6,59 & 10,34 & 11,01 & 9,76 & 24,50 \\
\hline
\end{tabular}

$\mathrm{MC}=$ massa corporal; $\mathrm{DBC}=$ diâmetro bi-acromial; DTS = diâmetros tóraco-sagital;

$\mathrm{PP}=$ perímetro do peito; ASTT = área de secção transversa do tronco.

O modelo para a totalidade da amostra $\left(\mathrm{F}_{2,75}=\right.$ 17,143; $\mathrm{P}<0,001)$ permitiu a entrada das variáveis perímetro peitoral $(\mathrm{t}=2,963$; $\mathrm{P}<0,001)$ e diâmetro tóraco-sagital ( $\mathrm{t}=2,333 ; \mathrm{P}=0,02)$, A equação preditiva da ASTT foi $\left(\mathrm{R}^{2}=0,32 ; \mathrm{Ra}^{2}=0,30 ; \mathrm{s}=\right.$ $158,93 ; \mathrm{P}<0,01)$ :

ASTT $-6,662 \cdot P P+17,019 \cdot D T S-210,708$

Para o subgrupo experts, o modelo final de regressão $\left(\mathrm{F}_{2,27}=6,078 ; \mathrm{P}=0,01\right)$ permitiu a entrada das variáveis perímetro peitoral $(\mathrm{t}=2,307 ; \mathrm{P}=0,03)$ e diâmetro tóraco-sagital $(t=1,858 ; \mathrm{P}=0,08)$. A equação preditiva da ASTT foi $\left(\mathrm{R}^{2}=0,33 ; \mathrm{Ra}^{2}=\right.$ $0,27 ; \mathrm{s}=165,41 ; \mathrm{P}<0,01)$

ASTT $-19,216 \cdot D T S+10,505 \cdot P P$

Para o subgrupo não experts, o modelo final de regressão $\left(\mathrm{F}_{2,47}=20,509 ; \mathrm{P}<0,001\right)$ incluiu as variáveis perímetro peitoral $(\mathrm{t}=1,050 ; \mathrm{P}=0,30)$ e diâmetro tóraco-sagital $(\mathrm{t}=1,606 ; \mathrm{P}=0,11)$. A equação preditiva da ASTT foi $\left(\mathrm{R}^{2}=0,48 ; \mathrm{Ra}^{2}=\right.$ $0,45 ; \mathrm{s}=136,89 ; \mathrm{P}<0,01)$ :

$A S T T-30,453 \cdot D T S+5,030 \cdot P P$

Quando se recorreu à análise da totalidade da amostra, mas com distinção do nível competitivo com base na variável dummy $(0=n \tilde{a} 0$ expert; $1=$ expert), o modelo de regressão ( $\mathrm{F} 3,75=17,001 ; \mathrm{P}<$ $0,001)$ permitiu a entrada das variáveis perímetro peitoral $(\mathrm{t}=3,253$; $\mathrm{P}=0,002)$ e diâmetro tóraco-sagital ( $\mathrm{t}=2,443 ; \mathrm{P}=0,017)$. A equação preditiva da ASTT foi $\left(\mathrm{R}^{2}=0,42 ; \mathrm{Ra}^{2}=0,39 ; \mathrm{s}=146,39 ; \mathrm{P}<0,01\right)$ :

ASTT $-19,984 \cdot$ DTS $+8,413 \cdot P P+19,851 \cdot$ Nivel $-414,695$

A figura 2 apresenta: (i) a comparação de valores médios entre a ASTT avaliada e estimada; (ii) o scatter gram da análise de regressão entre a ASTT avaliada e estimada; (iii) análise de Bland Altman entre a ASTT avaliada e ASTT estimada para a totalidade da amostra, esta com distinção do 

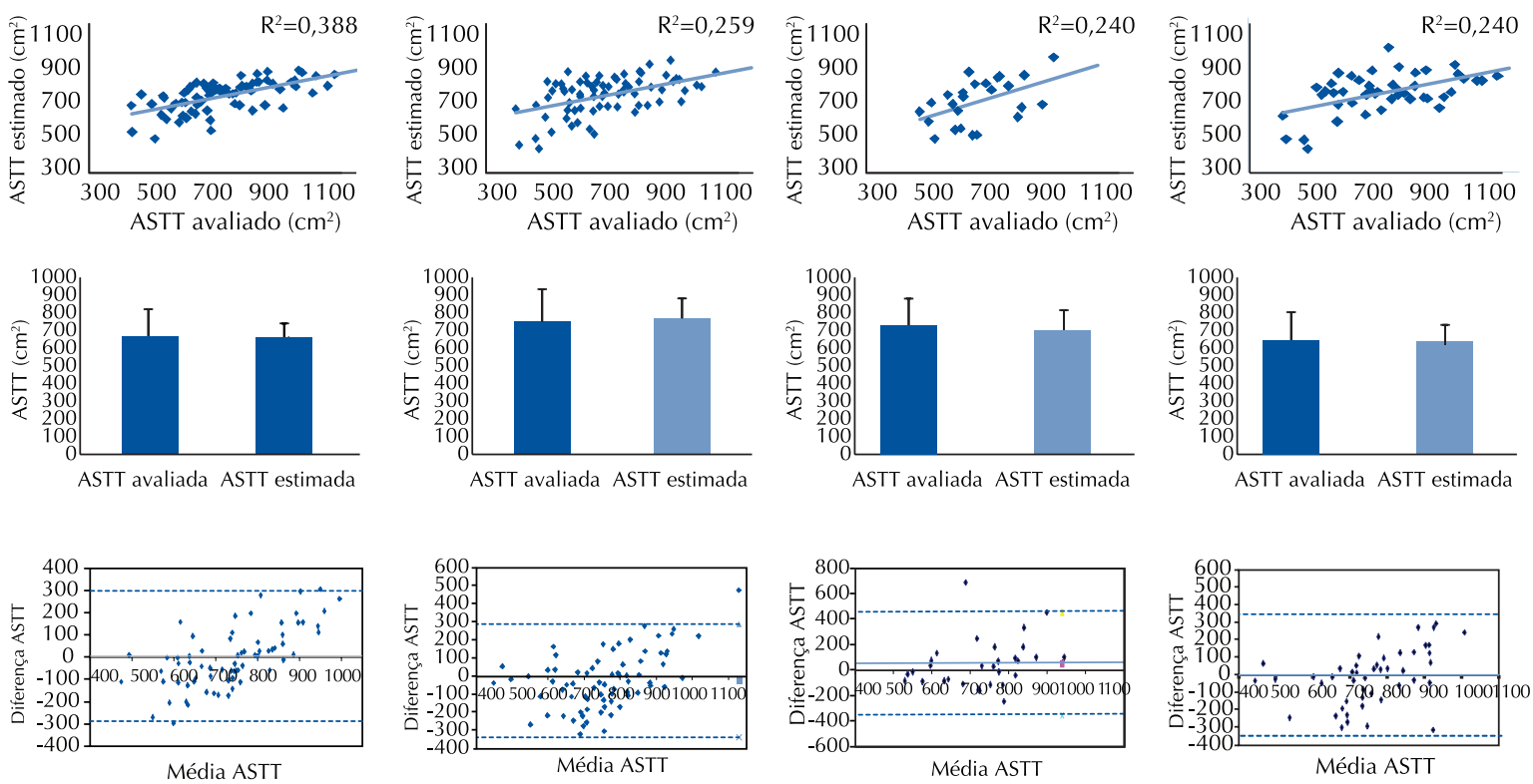

Figura 2. Comparação de valores médios, scatter gram e análise de Bland Altman entre as ASTT medida e estimada para a totalidade da amostra sem e com variável dummy, e para os subgrupos experts e não experts.

nível competitivo com base numa variável dummy, e para os subgrupos experts e não experts. Para todas as condições estudadas, não se verificaram diferenças estatisticamente significativas entre a ASTT avaliada e a estimada $(\mathrm{P}>0,05)$. Em todas as condições, a análise de regressão linear entre a ASTT avaliada e a estimada foi significativa. O ajuste para a totalidade da amostra $\left(\mathrm{R}^{2}=0,39\right)$, para os subgrupos masculino experts $\left(R^{2}=0,24\right)$, não experts $\left(\mathrm{R}^{2}=0,23\right)$ e para a totalidade da amostra com inclusão da variável dummy $\left(\mathrm{R}^{2}=\right.$ 0,26), foram consideradas moderadas. O critério da análise de Bland Altman foi respeitado para todas as condições estudadas. Ou seja, mais de 80 $\%$ dos plots encontraram-se dentro dos intervalos de confiança de $95 \%$,

\section{DISCUSSÃO}

Foi objectivo deste estudo desenvolver e validar equações preditivas da área de secção transversa do tronco humano. Verificou-se que os modelos desenvolvidos predizem de forma válida e significativa a ASTT no sexo masculino e de acordo com o nível competitivo. Foi utilizada uma grande amplitude de idades de forma a incluir na amostra um elevado número de sujeitos, visto estar-se restrito a praticantes de NPD, permitindo, ainda, que os modelos desenvolvidos possam ser utilizados por um número alargado de potenciais utilizadores.

Em relação à comparação dos valores médios da variável ASTT, o estudo de Clarys ${ }^{8}$ registrou um valor médio de ASTT de 767,33 \pm 92,51 [ $\left.\mathrm{cm}^{2}\right]$ para os estudantes de Educação Física e de 766,66 $\pm 123,88\left[\mathrm{~cm}^{2}\right]$ para os nadadores olímpicos holandeses. No estudo de Caspersen et al..$^{17}$, os autores também avaliaram a ASTT, registrando um valor médio de $890 \pm 60\left[\mathrm{~cm}^{2}\right]$. No presente estudo, a ASTT para a totalidade da amostra registrou um valor médio de 747,46 $\pm 184,59\left[\mathrm{~cm}^{2}\right]$ e de 715,57 \pm $175,51\left[\mathrm{~cm}^{2}\right]$ e $768,48 \pm 188,34\left[\mathrm{~cm}^{2}\right]$ para os subgrupos experts e não experts, respectivamente. Através da comparação dos estudos descritos, constata-se que os valores de ASTT registrados podem ser considerados semelhantes, muito embora ligeiramente inferiores. Esta diferença pode relacionar-se com a elevada amplitude de idades utilizada no presente estudo, sendo avaliados sujeitos entre os 10 e os 32 anos de idade.

As equações desenvolvidas foram consideradas significativas e com um nível qualitativo de predição tido como moderado $\left(0,32 \leq \mathrm{R}^{2} \leq 0,48 ; \mathrm{P}<\right.$ $0,01)$. Clarys ${ }^{8}$ desenvolveu a equação preditiva de ASTT, tendo considerado no modelo final a massa corporal e a estatura $\left(\mathrm{R}^{2}=0,50\right)$. Um outro estudo não descreveu associações significativas entre a ASTT e os diâmetros corporais avaliados ${ }^{18}$. Esta ausência de correlações significativas pode-se dever à reduzida dimensão da amostra avaliada. Para o presente estudo, considerou-se que outras variáveis, para além da massa corporal e estatura, pudessem ter um nível de predição superior para a estimação da ASTT. Em todas as condições, os modelos finais de estimação da ASTT incluíram a entrada 
das variáveis DTS e PP. Comparando os modelos preditivos do presente estudo com o de Clarys ${ }^{8}$, constata-se uma inferior capacidade preditiva. Tal facto reside, mais uma vez, na utilização de uma elevada amplitude de idades.

Na literatura, não existe consenso em relação aos procedimentos a utilizar para a validação de modelos preditivos. Há quem não sugira a utilização dos coeficientes de determinação e correlação para a validação de técnicas ou variáveis estimadas ${ }^{15}$. Outros consideram que o plot de Bland Altman é susceptível de evidenciar um sistemático viés de proporção ${ }^{19}$. Assim, foram utilizados três processos para validar os modelos desenvolvidos em que: (i) para todas as condições estudadas, não se verificaram diferenças estatisticamente significativas entre os valores médios da ASTT avaliada e estimada ( $\mathrm{p}$ $<0,05$ ); (ii) as regressões lineares simples entre a ASTT avaliada e medida, para todas as condições, foram significativas; (iii) todos os modelos respeitaram o critério de validade, em que mais de $80 \%$ dos plots de Bland Altman, entre a ASTT avaliada e estimada, se encontraram dentro dos $95 \%$ do intervalo de confiança.

Assim, este estudo apresenta a importância da estimação da variável ASTT, determinante na avaliação do arrasto passivo e activo, que são factores determinantes para performance. De um ponto de vista prático e aplicativo, ressalva-se: (i) a capacidade de avaliações técnicas de forma a reduzir o arrasto com o consequente aumento da performance, e não apenas através do aumento da propulsão; (ii) a utilização destas equações preditivas para a avaliação do arrasto passivo e activo, contribuindo com informações relevantes para treinadores, atletas e investigadores ${ }^{20}$.

O presente estudo apresenta como principais limitações: (i) os modelos preditivos não são válidos para o sexo feminino; (ii) os modelos desenvolvidos não são válidos para outras faixas etárias que não as estudadas; (ii) a consideração de mais variáveis nos modelos pode aumentar o nível de estimação da ASTT, mas dificultam e tornam mais moroso o processo de recolha e análise de dados; (iii) a ASTT não representa a área frontal de projecção do sujeito na posição horizontal, especialmente no caso de ocorrer um desalinhamento horizontal do corpo.

\section{CONCLUSÕES}

Pode concluir-se que: (i) todos os modelos preditivos desenvolvidos foram considerados significativos; (ii) todos os critérios nos vários procedimentos para validação dos modelos foram cumpridos. Logo, foram desenvolvidos e validados modelos de estimação da ASTT, para o sexo masculino, independentemente do nível competitivo ou tomando este em consideração.

\section{REFERÊNCIAS BIBLIOGRÁFICAS}

1. Kjendlie PL, Stallman RK. Drag characteristics of competitve swimming children and adults. J Appl Biomech 2008;24(1):35-42.

2. Vilas-Boas JP. Aproximação biofísica ao desempenho e ao treino de nadadores. Rev, Paul Educ Fís 2000;14(2):107-17.

3. Maglischo EW. Swimming even faster. Mountainview: Mayfield Publishing Company; 1993.

4. Marinho D, Barbosa T, Costa M, Figueiredo C, Reis $\mathrm{V}$, Silva A, et al. Can 8-weeks of training affect active drag in young swimmers? J Sports Sci Med 2010;9:71-8.

5. Costill DL, Maglischo EW, Richardson AB. Swimming. Oxford: Blackwell Scientific Publications; 1992.

6. Pendergast DR, Capelli C, Craig AB, di Prampero PE, Minetti AE, Mollendorf J, et al. Biophysics in swimming. Rev Port Cien Desp 2006;6(Supl 2):185-9.

7. Kolmogorov S, Rumyantseva O, Gordon B, Cappaert J. Hydrodynamic characteristics of competitive swimmers of different genders and performance levels. J Appl Biomech 1997;13(1):88-97.

8. Clarys J. Human morphology and hydrodynamics. In. Terauds J, Beddingfield W, organizadores. Swimming III. Baltimore: University Parck Press; 1979. p, 3-41.

9. Barbosa TM, Costa MJ, Marques MC, Silva AJ, Marinho DA. A model for active drag force exogenous variables in young swimmers. Med Sci Sports Exerc 2010;42(5):687-8.

10. Prestes J, Leite R, Leite G, Donatto F, Urtado C, Bartolomeu Neto J, et al. Características antropométricas de jovens nadadores brasileiros do sexo masculino e feminino em diferentes categorias competitivas. Rev Bras Cinenatropom Desempenho Hum 2006;8(4):25-31.

11. Mazza J, Ackland TR, Bach T, Cosolito P, Absolute body size. In: Carter L, Ackland TR, organizadores. Kineanthropometry in Aquatic Sports. Champaign: Human kinetics; 1994. p, 15-54.

12. Barbosa TM, Fernandes RJ, Morouço P, Vilas-Boas JP. Predicting the intra-cyclic variation of the velocity of the centre of mass from segmental velocities in butterfly stroke: a pilot study. J Sports Sci Med 2008;7:201-9.

13. Wolfram U, Wilke HJ, Zysset PK. Valid micro finite element models of vertebral trabecular bone can be obtained using tissue properties measured with nanoindentation under wet conditions. J Biomech 2010;43:1731-7.

14. Baldari C, Bonavolontà V, Emerenziani GP, Gallotta MC, Silva AJ, Guidetti L, Accuracy, reliability, linearity of Accutrend and Lactate Pro versus EBIO plus analyzer. Eur J Appl Physiol 2009;107:105-11. 
15. Kristensen MT, Bandholm T, Holm B, Ekdahl C, Kehlet H, Timed up \& go test score in patients with hip fracture is related to the type of walking aid. Arch Phys Med Rehabil 2009;90:1760-5.

16. Bland JM, Altman DG. Statistical method for assessing agreement between two methods of clinical measurement. Lancet i 1986;307-10.

17. Caspersen C, Berthelsen PA, Eik M, Pâkozdi C, Kjendlie PL. Added mass in human swimmers: age and gender differences. J Biomech 2010;43:2369-73.

18. Huijing P, Toussaint H, Mackay R, Vervoon K, Clarys J, Hollander AP. Active drag related to body dimensions. In. Ungerechts B, Wilke K, Reischle K, organizadores. Swimming Science V. Champaign: Human Kinetics Books; 1988. p, 31-37.

19. Hopkins WG. Bias in Bland-Altman but not regression validity analyses. Sportscience 2004;8:42-46.
20. Vilas-Boas JP, Costa L, Fernandes RJ, Ribeiro J, Figueiredo P, Marinho D, et al. Determination of the drag coeficiente during the first and second gliding positions of the breaststoke underwater stroke. J Appl Biomech 2010;26:324-31.

Endereço para correspondência Jorge Estrela Morais Instituto Politécnico de Bragança Campus de Santa Apolónia Apartado 1038 5301-854 - Braguança, Portugal E-mail: morais.jorgestrela@gmail.com 